\title{
An Optimized Approach for Image Segmentation on Mobile Devices
}

\author{
Hemantkumar R. Turkar ${ }^{1}$,Gopal Sakarkar ${ }^{2}$ \\ ${ }^{1}$ Rajiv Gandhi College of Engineering \& Research, Nagpur, India \\ ${ }^{2} \mathrm{G}$ H Raisoni College of Engineering , Nagpur
}

Article History: Received: 11 January 2021; Accepted: 27 February 2021; Published online: 5 April 2021

\begin{abstract}
Now a day's image segmentation is widely used in many multimedia applications. We have introduced the optimized approach for image segmentation based on clustering for use on smart devices. The proposed optimized approach is based on the combination of partitioning of images using quad-tree and Ant Colony Optimization. This approach utilizes the strong ability of ACO i.e global optimization. The proposed optimized algorithm is evaluated on images of standard data set and its performance is compared with existing clustering algorithms. The qualitative and quantitative analysis has been performed to measure the efficacy of the optimized approach over conventional existing algorithms. This procedure obtains better quality results than existing clustering algorithms.
\end{abstract}

Keywords: Image segmentation; optimization; quad tree; ACO

\section{Introduction}

An optimization algorithm is a process of executing and comparing various solutions until an optimum solution is found. Optimization approaches are divided into classical optimization techniques and non-classical optimization techniques. A classical optimization technique has various methods such as direct method, gradient method, linear programming method and interior point method. Optimization is a technique which obtains the best solution to satisfy certain objectives. Design of various engineering problems is very complex process. Formulation of such engineering problems with an objective to satisfy all conditions of design is possible by proper use of various optimization techniques.

The classical optimization techniques are beneficial for finding the ideal solution of uninterrupted and differentiable tasks. These techniques are used in analytical methods for differential calculus in locating the ideal solution. Classical optimization techniques handled various problems such as single variable functions, multivariable functions without constraints and with equality \& inequality constraints.

The Gradient based optimization techniques are more efficient with considering gradient information. These types of method use the calculus and derivatives of the objectives. The various types of gradient-based methods are steepest decent method, Newton's method, Conjugate gradient method etc.

Direct method of classical optimization uses to reduce the variational problem to ordinary limitation problem by determining the limitation of a function of several variables. Linear programming is a powerful tool for solving the problems in real world modeling.

Interior methods are fast and their influence has changed both the theory and practice of constrained optimization. This method contains the universal conspicuous feature of the constrained optimization which uses for landscape today.

Non classical optimization technique has various methods such as Ant Colony Optimization, Genetic Algorithms, Particle Swarm Optimization algorithms etc. The optimization techniques have wide range of applications in medical field to detect abnormalities, face detection in criminal purpose, tumour detection etc. As a result, optimization techniques can be utilized to generate an optimum outcome in comparison with simple segmentation method. There are several optimization techniques on image segmentation, all with the goal of defining the best solution.

The rest of the paper is organized in the following manner: Section 2 introduces optimization techniques. Section 3 explains the optimized approach and section 4 summarizes the experimental results.

\section{Optimization Techniques}

Optimized $\mathrm{k}$-means algorithm can homogeneously divide an image into sections of interest with the ability of escaping the dead centre and trapped centre at local minima phenomena [1]. The alteration on the hard 
membership theory as engaged by the formal k-means algorithm is measured. As the process of a pixel is assigned to its subordinate cluster, if the pixel has two or more adjoining cluster centers with same distance, the pixel is assigned to the cluster with null or a smaller fitness value. Easy method for diagnosing tumour in brain magnetic resonance imaging [2]. The tumor is an uninhibited multiplication of cells of body tissues. The main issue in treatment of brain tumour is inaccurate detection of the tumour. Depending on the characteristics of tumour the line of treatment varies. It is very difficult to identify the tumour in human body manually. To overcome the above problem our paper suggests an approach for finding of brain tumor using k-means and improved fuzzy C-means for image segmentation.

Optimization method which segments the image of pulmonary cancer [3]. Two methods such as Fuzzy kmeans clustering method and Particle Swarm optimization method are instigated for partition of image. The numerous parameters like PSNR and MSE are evaluated. Optimum solution is given by the proposed method. Various meta-heuristic methods for segmentation of brain tumour in 2D magnetic resonance imaging [4] by using Ant Colony Optimization (ACO), Genetic Algorithm (GA) and Particle Swarm Optimization (PSO). It is proposed that PSO algorithm out performs over ACO and GA algorithms. The enactment of Fuzzy C-means combined with Particle Swarm optimization method [5] and its variants are studied in various application arenas. To analyse methods in various arenas many metrics are utilize such as global consistency errors and deviation of information is used. The integrated approach proposed in this paper performs with better accuracy as compared to other techniques.

A wide overview of bio-inspired optimization algorithms [6], grouped by the various biological fields that inspired each and the areas where these algorithms have been most successfully applied. Almost all the algorithms execute with heuristic population-based search processes that combine random variation and selection. An image clustering algorithm using Particle Swarm Optimization (PSO) with two improved fitness function [7]. Experimental analysis shows that clustering approach based on PSO performs better than the Kmeans by generating more close clusters and larger inter-cluster separations.

Hybrid techniques using various PSO trial-and-errors to enhance the k-means method and investigate the consistency of parametric value for various variants of PSO and k-means algorithm [8]. The k-means algorithm is made more stable by applying PSO for finding improved solutions.

Genetic Algorithm is an investigative technique useful for optimizing combinative problem. In computer science genetic algorithm is a meta heuristic procedure based on the Darwins theory of evolution. It is widely popular for its usage to generate high quality solution to optimization. It relies on bio-inspired operators such as mutation selection and crossover. The basic steps in genetic algorithm are selection of survival of the fitness, mating between individuals and lastly mutation which presents random Modification. The best chromosomes in genetic algorithm approach are selected into the mating pool. In mating pool chromosomes undergo cross over and mutation to produce new set of solutions.

The PSO method is based on swarms like flocks of bird schools of fish. Individuals interact with each other during their growth and group members. In a PSO system the flight of each particle adjusts itself using its own experience as well as adjacent particle experience. This leads to encounter the best position by itself and its neighbours. There are various advantages of PSO such as it is very simple and fast, easier to implement, more efficient in maintaining the diversity.

\section{Optimized Approach for Image Segmentation}

For development of optimized approach of color and grey level image segmentation on mobile devices the quad tree partitioning and ant colony optimization algorithm is used.

\section{Partitioning of image using quad tree}

In quad-tree partition method the image is denoted by layers in a structure of a tree. The root node of the tree is labeled by $C$ (entire Image) and the children of the root nodes are the four quadrant of the entire image/root node shown in figure 3.1. The process is carried out until there are no more regions to divide. Recursively calling this process leads to generate the quad-tree structure as a tree for representation of an image. Quad tree based image segmentation provides accuracy and efficacy which reduces the cost of reconstruction significantly [9]. The quad-tree is executed at each of the red, green, and blue channels of RGB color space. This will divide four equal-size sub-blocks. Quad tree processes the whole image into pixels [10]. 


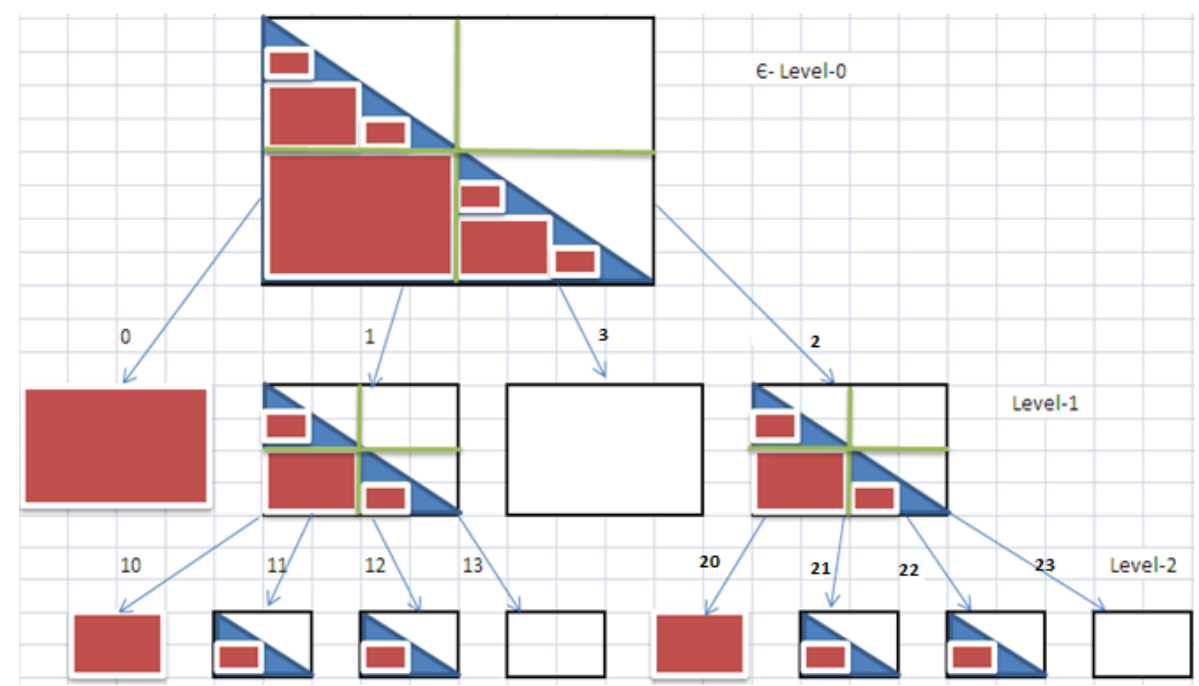

Figure 3.1: Quad-tree Representation of Image

\section{Optimization using ACO}

In this approach the optimization technique is used. It is a modern demographic approach is motivated by the surveillance of real ant colony activities. Real ants are having ability to find shortest possible path that to a straight line. An ant secretes a chemical substance called Pheromone during walking and each ant follows the path way having Pheromone. Process of minimum residents of ants formed with stochastic iterative process. In this process every ant helps in solving the problem. So that every ant contributes a part in solving the entire problem. Flow of basic algorithm of ACO is shown in Figure 3.2.

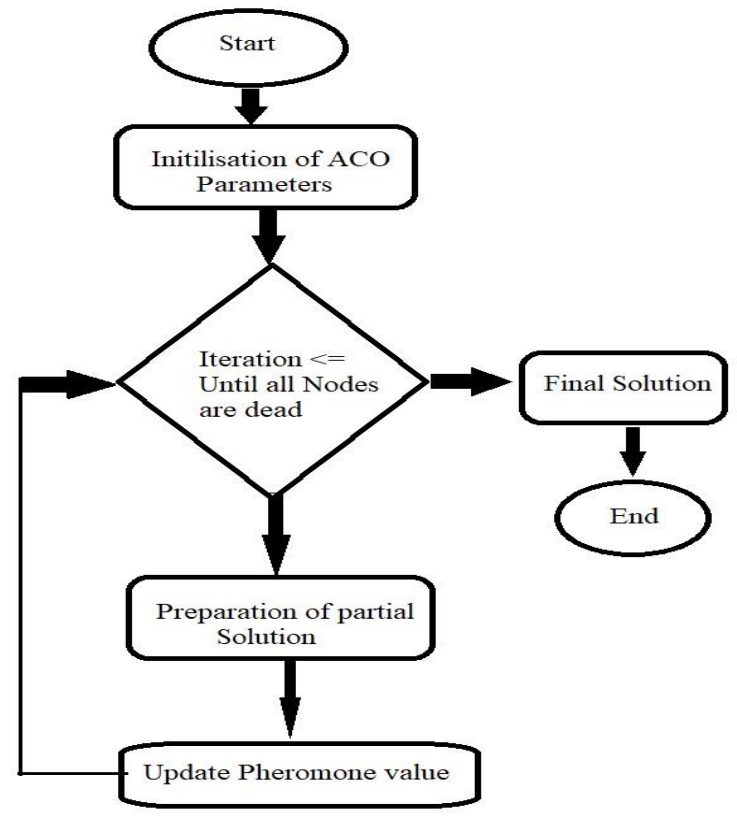

Figure 3.2: Flow of basic algorithm of ACO

\section{Experimental Results of Optimized Approach on RGB color space}

The comparison of implemented approaches is carried out for MSE, PSNR and MAE. Each parameter is evaluated for developed KM, MKM, EMKM and Optimized Approach (Proposed Approach-PA) for RGB images having cluster count of $\mathrm{k}$, in which $\mathrm{k}$ is equal to 3, 4 and 5 correspondingly. Table 4.1, Table 4.2, and Table 4.3 shows the summary of MSE, PSNR and MAE evaluation for the RGB color space. 


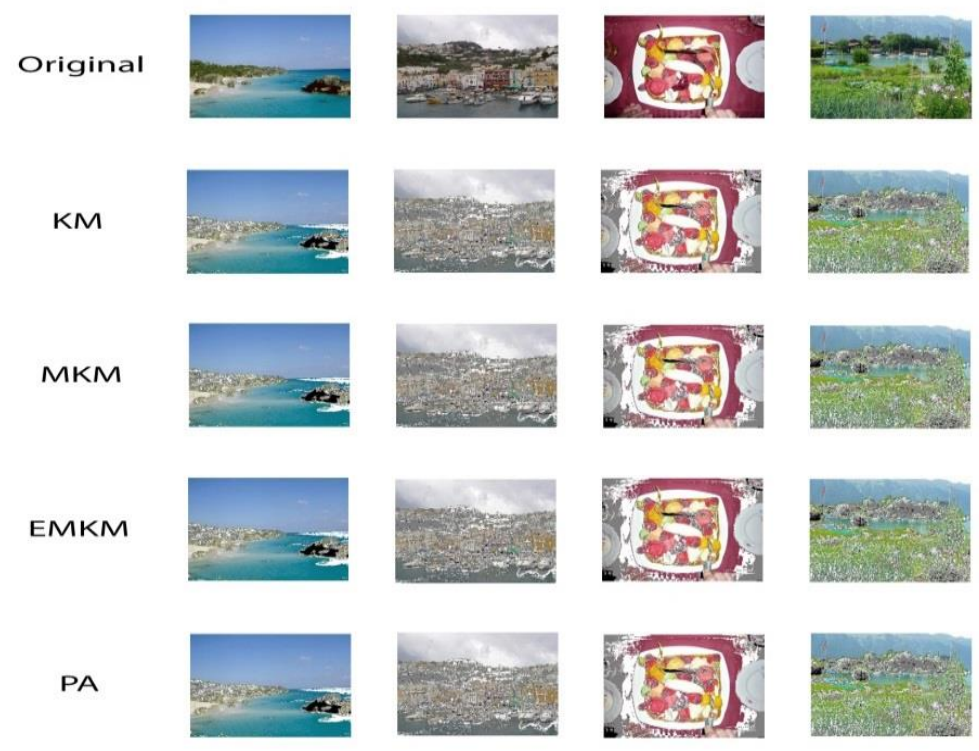

Figure 4.1: Segmentation of RGB images for cluster value $\mathrm{K}=3$

Table 4.1: MSE Evaluation of Approaches for selected RGB images

\begin{tabular}{|c|c|c|c|c|}
\hline \multirow{2}{*}{\multicolumn{2}{|c|}{$\begin{array}{c}\text { RGB Color Snace } \\
\text { Cluster }\end{array}$}} & \multicolumn{3}{|c|}{ Mean Saware Frror (MSE) } \\
\hline & & $k=3$ & $k=4$ & $k=5$ \\
\hline \multirow{4}{*}{ KM } & Image 1 & 25310.62 & 36285.32 & 6946.82 \\
\hline & Image 2 & 10394 & 36319.74 & 23143.33 \\
\hline & Image 3 & 2269.57 & 34153.26 & 28094.96 \\
\hline & Image 4 & 3187385 & 100441 & 2495488 \\
\hline \multirow{4}{*}{ MKM } & Image 1 & 24490.08 & 36285.32 & 694682 \\
\hline & Image 2 & 1015752 & 3631974 & 2314333 \\
\hline & Image 3 & 1698.97 & 34153.26 & 28094.96 \\
\hline & Image 4 & 31357.56 & 1004.41 & 24954.88 \\
\hline \multirow{4}{*}{ EMKM } & Image 1 & 25310.62 & 36285.32 & 6946.82 \\
\hline & Image 2 & 910884 & 36319.74 & 23143.33 \\
\hline & Image 3 & 91655 & 3415326 & 2809496 \\
\hline & Image 4 & 30505.42 & 1004.41 & 24954.88 \\
\hline \multirow{4}{*}{ PA } & Image 1 & 23762.7 & 36285.32 & 6946.82 \\
\hline & Image 2 & 8507.84 & 36319.74 & 23143.33 \\
\hline & Image 3 & 498.99 & 34153.26 & 28094.96 \\
\hline & Image 4 & 30250.32 & 1004.41 & 24954.88 \\
\hline
\end{tabular}

Table 4.2: PSNR Evaluation of Approaches for selected RGB images

\begin{tabular}{|c|c|c|c|c|}
\hline \multicolumn{2}{|c|}{ RGB Color Space } & \multicolumn{3}{c|}{ PSNR } \\
\hline \multirow{3}{*}{ Cluster } & $\mathrm{k}=3$ & $\mathrm{k}=4$ & $\mathrm{k}=5$ \\
\hline \multirow{4}{*}{ KM } & Image 1 & 4.09 & 2.53 & 9.71 \\
\cline { 2 - 5 } & Image 2 & 7.96 & 2.52 & 4.48 \\
\cline { 2 - 5 } & Image 3 & 14.57 & 2.79 & 3.64 \\
\cline { 2 - 5 } & Image 4 & 3.09 & 18.11 & 4.15 \\
\hline \multirow{4}{*}{ MKM } & Image 1 & 4.24 & 2.53 & 9.71 \\
\cline { 2 - 5 } & Image 2 & 8.06 & 2.52 & 4.48 \\
\cline { 2 - 5 } & Image 3 & 15.82 & 2.79 & 3.64 \\
\cline { 2 - 5 } & Image 4 & 3.16 & 18.11 & 4.15 \\
\hline \multirow{4}{*}{ EMKM } & Image 1 & 4.09 & 2.53 & 9.71 \\
\cline { 2 - 5 } & Image 2 & 8.53 & 2.52 & 4.48 \\
\cline { 2 - 5 } & Image 3 & 18.5 & 2.79 & 3.64 \\
\cline { 2 - 5 } & Image 4 & 3.28 & 18.11 & 4.15 \\
\hline \multirow{4}{*}{ PA } & Image 1 & 4.37 & 2.53 & 9.71 \\
\cline { 2 - 5 } & Image 2 & 8.83 & 2.52 & 4.48 \\
\cline { 2 - 5 } & Image 3 & 21.14 & 2.79 & 3.64 \\
\cline { 2 - 5 } & Image 4 & 3.32 & 18.11 & 4.15 \\
\hline
\end{tabular}


Table 4.3: MAE Evaluation of Approaches for selected RGB images

\begin{tabular}{|c|c|c|c|c|}
\hline \multicolumn{2}{|c|}{ RGB Color Space } & \multicolumn{2}{c|}{ MAE } & $\mathrm{k}=5$ \\
\hline \multirow{3}{*}{ Cluster } & $\mathrm{k}=3$ & $\mathrm{k}=4$ & 0.12 \\
\hline \multirow{4}{*}{ KM } & Image 1 & 0.44 & 0.63 & 0.4 \\
\cline { 2 - 5 } & Image 2 & 0.18 & 0.63 & 0.48 \\
\cline { 2 - 5 } & Image 3 & 0.03 & 0.59 & 0.43 \\
\cline { 2 - 5 } & Image 4 & 0.55 & 0.01 & 0.12 \\
\hline \multirow{4}{*}{ MKM } & Image 1 & 0.42 & 0.63 & 0.4 \\
\cline { 2 - 5 } & Image 2 & 0.17 & 0.63 & 0.48 \\
\cline { 2 - 5 } & Image 3 & 0.02 & 0.59 & 0.43 \\
\hline \multirow{4}{*}{ EMKM } & Image 4 & 0.54 & 0.01 & 0.12 \\
\cline { 2 - 5 } & Image 1 & 0.44 & 0.63 & 0.4 \\
\cline { 2 - 5 } & Image 2 & 0.15 & 0.63 & 0.48 \\
\cline { 2 - 5 } & Image 3 & 0.01 & 0.59 & 0.43 \\
\hline \multirow{4}{*}{ PA } & Image 4 & 0.53 & 0.01 & 0.12 \\
\cline { 2 - 5 } & Image 1 & 0.41 & 0.63 & 0.4 \\
\cline { 2 - 5 } & Image 2 & 0.14 & 0.63 & 0.48 \\
\cline { 2 - 5 } & Image 3 & 0.01 & 0.59 & 0.43 \\
\hline
\end{tabular}

Graphical representation of the attained results of MSE evaluation of developed KM, MKM, EMKM and Optimized Approach with k=3 on RGB color space is shown in Figure 4.2. From the graphical representation it is evident that the optimized approach gives improved image segmentation with less MSE value for $\mathrm{K}=3$ as compared to other clustering based image segmentation approaches.

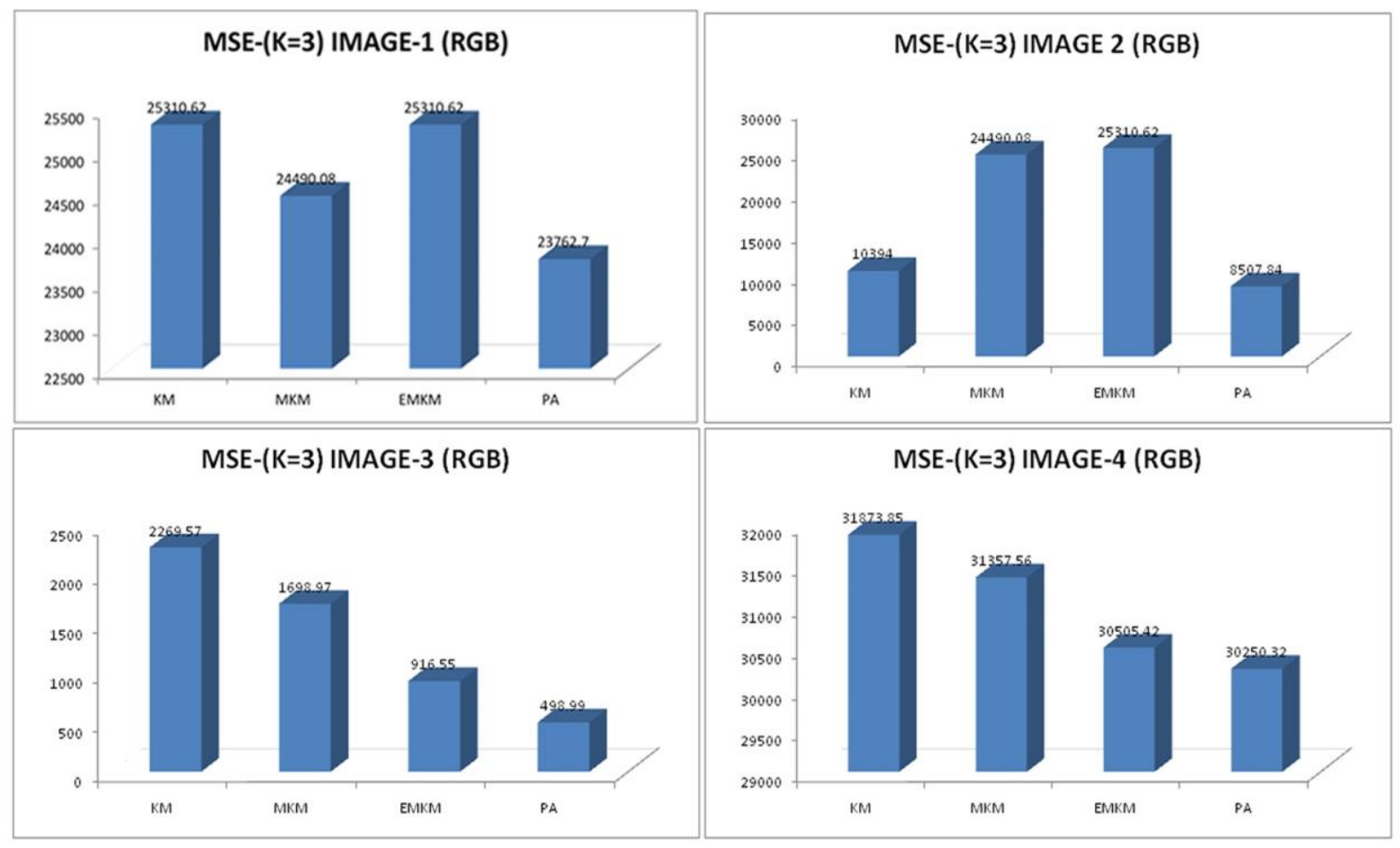

Figure 4.2: MSE evaluation of KM, MKM, EMKM and PA with k=3 for RGB

Graphical representation of the attained results of PSNR evaluation of developed KM, MKM, EMKM and Optimized Approach with k=3 on RGB color space is shown in Figure 4.3. From the graphical representation it is evident that the optimized approach gives better image segmentation with less PSNR value for $\mathrm{K}=3$ as compared to other clustering based image segmentation. 

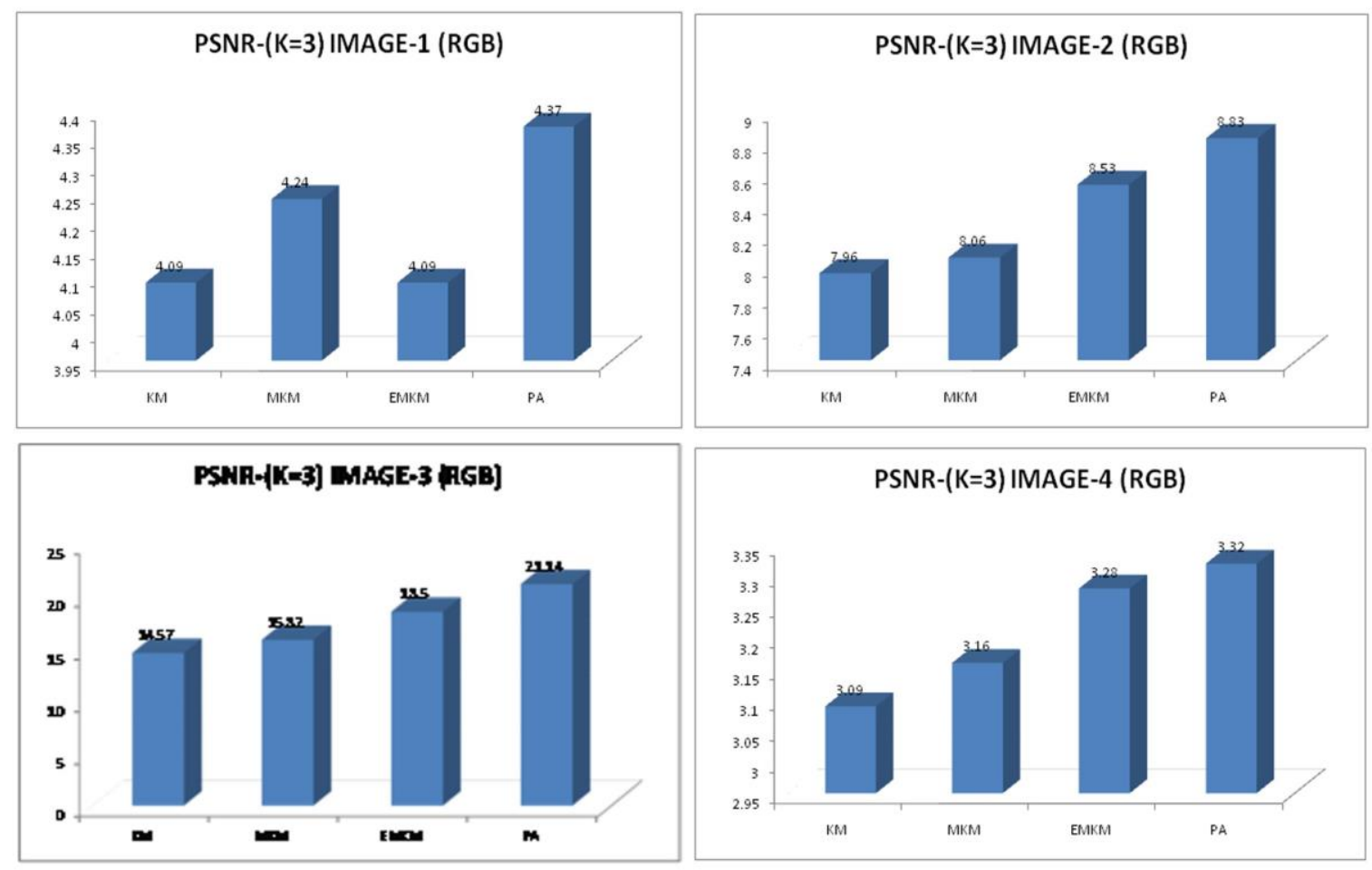

Figure 4.3: PSNR evaluation of KM, MKM, EMKM and PA with k=3 for RGB

Graphical representation of the attained results of MAE evaluation of developed KM, MKM, EMKM and Optimized Approach with k=3 on RGB color space is shown in Figure 4.4. From the graphical representation it is evident that the optimized approach gives better image segmentation with less MAE value for $K=3$ as compared to other clustering based image segmentation approaches.

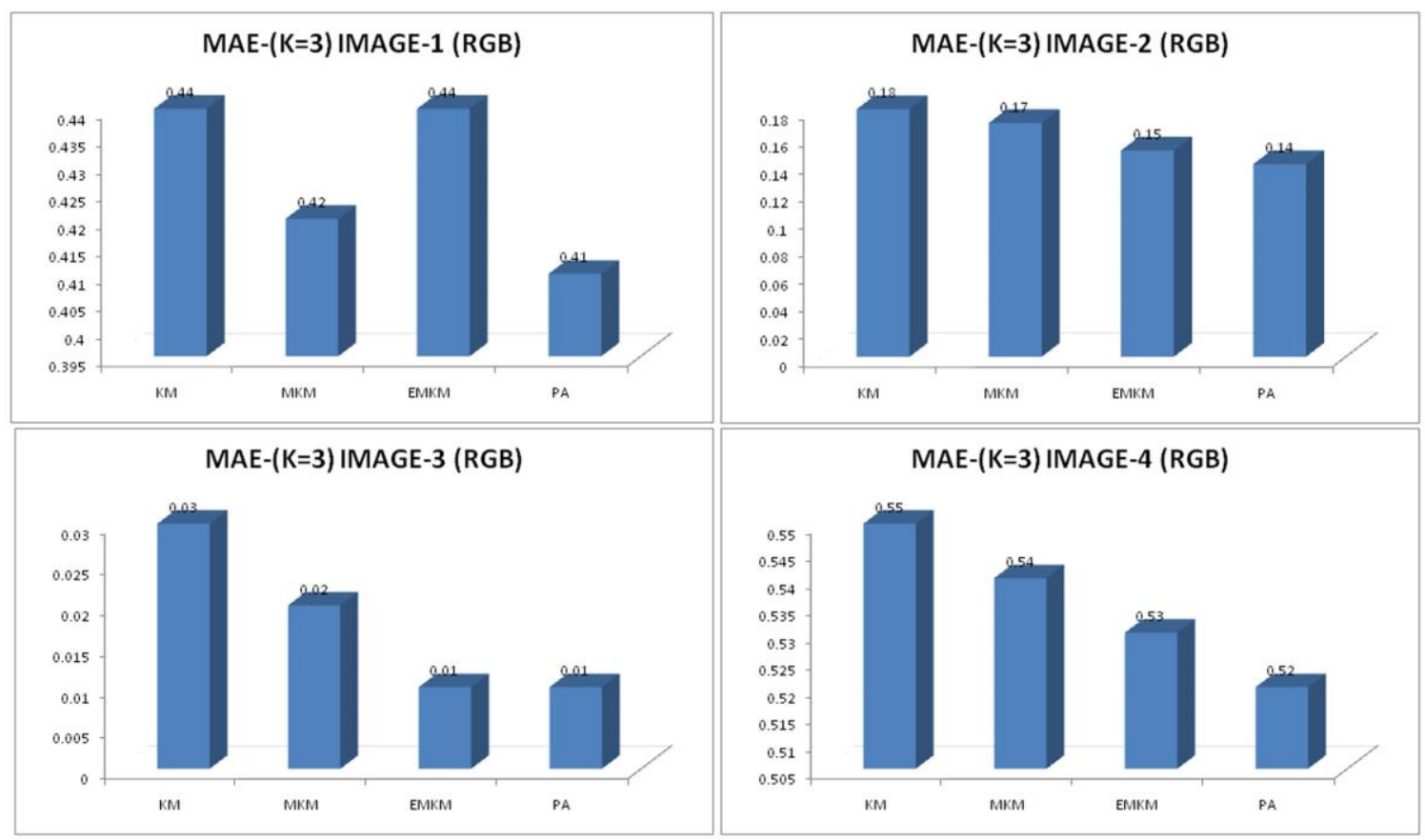

Figure 4.4: MAE evaluation of KM, MKM, EMKM and PA with k=3 for RGB 


\section{Conclusion and Future scope}

The proposed optimized approach based on quad tree and Ant Colony Optimization which determines the optimal clustering of an image data set which produced a better segmentation. Optimized approach has a good segmentation result in comparison with developed and implemented KM, MKM, EMKM approaches regardless the number of clusters used. Quantitative analysis discovers that the optimized approach produces better segmented images with the evaluation parameters namely MSE, MAE and PSNR. Qualitative analysis is carried out which proves that obtained segmentation results of optimized approach are better than the developed KM, MKM, EMKM approaches.

\section{References}

1. F.U. Siddiqui and N. A. Mat Isa, "Optimized K-means (OKM) clustering algorithm for image segmentation”, Opto-Electronics Review, volume 20, Issue 3, pp. 216-225, May 2012

2. Dr. A. J. Patil, Dr. Prerana Jain and Ashwini Pachpande, “Automatic Brain Tumor Detection Using KMeans and RFLICM", International Journal of Advanced Research in Electrical, Electronics and Instrumentation Engineering, ISSN (Online): 2278 8875, Volume 3, Issue 12, December 2014

3. Sharma, AMAN KUMAR, and A. N. J. U. Bala. "Maker Based Watershed Transformation for Image Segmentation." International Journal of Computer Science Engineering and Information Technology Research 3 (2013): 187-192.

4. R. Joel George, D. AnithaJebaKumari, "Segmentation and Analysis of Lung Cancer Images Using Optimization Technique", International Journal of Engineering and Innovative Technology (IJEIT) Volume 3, Issue 10, April 2014

5. Sharma, AMAN KUMAR, and A. N. J. U. Bala. "Maker Based Watershed Transformation for Image Segmentation." International Journal of Computer Science Engineering and Information Technology Research 3 (2013): 187-192.

6. M. C. Jobin Christ, S. Sivagowri and P. Ganesh Babu, "Segmentation of Brain Tumors using Meta Heuristic Algorithms", OPEN JOURNAL OF COMMUNICATIONS AND SOFTWARE, Volume 1, Number 1, May 2014

7. N. Gopi Raju and P. A. Nageswara Rao, "Particle Swarm Optimization Methods for Image Segmentation Applied In Mammography”, International Journal of Engineering Research and Applications, ISSN : 22489622, pp. 1572-1579, Volume 3, Issue 6, Nov-Dec 2013

8. Sivaperumal, S., and M. Sundhararajan. "Brain Tumor Analysis for MRI Image Segmentation using Seeded Region Growing and PCNN." International Journal of Electronics, Communication \& Instrumentation Engineering Research and Development (IJECIERD) 3.2 (2013): 175-182.

9. S. Binitha and S Siva Sathya, "A Survey of Bio inspired Optimization Algorithms", International Journal of Soft Computing and Engineering (IJSCE), ISSN: 2231-2307, Volume 2, Issue 2, May 2012

10. Man To Wong, H. Xiangjian and Wei-Chang Yeh, "Image Clustering Using Particle Swarm Optimization",Proceedings of the IEEE Congress on Evolutionary Computation, CEC 2011, New Orleans, LA, USA, 5-8 June 2011

11. Ingale, Mayur, and KS BHAGAT2\& PM MAHAJAN. "Image Segmentation Using Gabor Filter And Wavelet Transform." International Journal Of Electrical And Electronics Engineering Research (IJEEER) ISSN: 25-28.

12. Chih-Cheng Hung and Li Wan, "Hybridization of Particle Swarm Optimization with the K-Means Algorithm for Image Classification", IEEE Symposium on Computational Intelligence for Image Processing, pp. 978-1-4244-2760-4, May 2009

13. Patne, G., and P. Ghonge. "Automization of agriculture products defect detection and grading using image processing system." International Journal of Computer Science Engineering and Information Technology Research 8.3 (2018): 25-32.

14. F. Keissarian, “A New Quad-tree Segmented Image Compression Scheme using Histogram Analysis and Pattern Matching”, IEEE International Conference on Computer and Automation Engineering (ICCAE), Volume 5, 2010

15. Ken-Chung Ho and Chia-Lung Hung , "A Hue-Based Quad-Tree Method for Color Image Segmentation”, IEEE International Conference on Consumer Electronics-Taiwan (ICCETW), 2017

16. Khodasakr, Anuja, and Siddharth Ladhake. "Interactive Image Retrieval with Advanced Clustering Strategy." International Journal of Computer Science Engineering and Information Technology Research (IJCSEITR) 5.1, Feb 2015, 3542. 